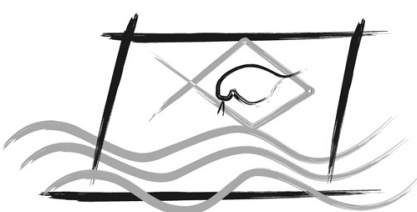

ECOTOX - BRASIL

\title{
A determinação da toxicidade crônica para peixes baseada apenas na sobrevivência é suficiente?
}

\author{
A. Arenzon ${ }^{1}$; C. De Lorenzo ${ }^{2}$; N.J. Coimbra ${ }^{3} \&$ U.H. Schulz \\ ${ }^{1}$ Universidade Federal do Rio Grande do Sul, Centro de Ecologia, Porto Alegre, RS, Brasil. \\ ${ }^{2}$ Universidade Federal do Rio Grande do Sul, Faculdade de Veterinária, Porto Alegre, RS, Brasil. \\ ${ }^{3}$ Ecotox Análise e Consultoria Ambiental Ltda, Porto Alegre, RS, Brasil. \\ ${ }^{4}$ Universidade do Vale do Rio dos Sinos, São Leopoldo, RS.
}

(Received April 26, 2013; Accept July 01, 2013)

\begin{abstract}
The determination of chronic toxicity based only in fish survival is enough? - In Brazil, the evaluation of short-term chronic effects on fish follows ABNT NBR 15499/2007. It can be conducted with two species: Danio rerio or Pimephales promelas. In the analysis with $P$. promelas, results are obtained by evaluating survival and growth of the organisms. However, when $D$. rerio is used, only the survival is evaluated. The objective of this paper is to assess whether the observed chronic toxicity on growth can always be extrapolated from the survival data, as suggested by ABNT NBR 15499/2007. 687 chronic tests with $P$. promelas were conducted. Three groups of results were observed: samples with toxicity effect only on growth, samples with toxicity effect only on survival and samples that showed toxicity effect on the survival and growth, simultaneously. The toxicity solely on the survival of organisms was observed in $56 \%$ and $31 \%$ of the tests for environmental samples and effluents, respectively. Analyzing the effluent tests where toxicity was detected on the survival, $32 \%$ also showed toxicity on growth. The toxic effect on the growth represented merely $44 \%$ of the environmental samples and $37 \%$ of the effluent samples. Therefore, chronic toxicity evaluations based exclusively on the survival of organisms may produce false negative results, especially when mortality is not detected at all.
\end{abstract}

Keywords: ABNT 15499/2007, chronic extrapolated value, Ecotoxicology, Pimephales promelas, Danio rerio.

\section{INTRODUÇÃO}

No ambiente aquático muitas vezes os organismos estão expostos a agentes químicos em níveis não letais podendo não levar à morte do organismo, mas causando distúrbios fisiológicos e/ou comportamentais a longo prazo (Zagatto \& Bertoletti, 2006). Esses efeitos são detectados em ensaios de toxicidade crônica, que permitem avaliar os efeitos adversos mais sutis.

A norma Brasileira ABNT-NBR 15499 (ABNT, 2007), que normatiza a avaliação dos efeitos crônicos de curta duração em peixes, traz na sua metodologia duas espécies: Pimephales promelas, conhecido como "fathead minnow" e Danio rerio, conhecido também por peixe zebra ou paulistinha. Para a avaliação realizada com $P$. promelas os resultados são obtidos avaliando a sobrevivência e o crescimento, em peso, dos organismos-teste, no período de exposição do ensaio. Todavia, para os ensaios com $D$. rerio, somente a sobrevivência pode ser avaliada devido a sua reduzida capacidade de ingestão do alimento de origem externa na fase larval e, conseqüentemente, no crescimento inexpressivo desse organismo. Desta forma, o método prevê que uma estimativa do efeito crônico sobre o crescimento seja obtida pela extrapolação de dados de sobrevivência. Para a avaliação do efeito crônico sobre o $D$.

*Corresponding author: Alexandre Arenzon; e-mail: alex@ufrgs.br 
rerio é, então, usado o calculo de VCest(I). Este método é descrito na norma ABNT-NBR 15499 (ABNT, 2007), que é a multiplicação da $\mathrm{CL}_{15}$ (Concentração letal inicial que causa efeito na sobrevivência de $15 \%$ dos organismos no tempo de exposição) pelo fator 0,3 (valor fixo), sendo assim o valor crônico estimado.

Desta forma, o objetivo proposto para o presente trabalho é avaliar, para diferentes tipos de amostras, a possibilidade do método apresentado na norma Brasileira ABNT-NBR 15499 não detectar efeitos tóxicos crônicos sobre $D$. rerio, quando esta for extrapolada exclusivamente com dados referentes à sobrevivência dos organismos.

\section{MATERIAIS E MÉTODOS}

Os resultados apresentados neste estudo são baseados na avaliação de ensaios realizados pelo Laboratório de Ecotoxicologia do Centro de Ecologia da Universidade Federal do Rio Grande do Sul (UFRGS), assim como pelo Laboratório Ecotox Análise e Consultoria Ambiental Ltda.. Foram utilizados dados provenientes de amostras ambientais (águas superficiais e subterrâneas), amostras de efluentes industriais, bem como substâncias de referência. No total foram tabulados os dados de 687 ensaios crônicos de curta duração com o peixe Pimephales promelas, todos executados segundo a norma ABNT-NBR 15499/2007.

Os dados foram tabulados separadamente para amostras ambientais (471 amostras), amostras de efluente (202 amostras) e para o cloreto de sódio $(\mathrm{NaCl})$ da marca Merck (14 amostras). Para cada tipo de amostra, aquelas consideradas tóxicas foram divididas em três grupos: amostras com efeitos somente na sobrevivência; amostras com efeitos sobre a sobrevivência e sobre o crescimento; e amostras com toxicidade exclusivamente sobre o crescimento. Foram consideradas tóxicas aquelas amostras cujos efeitos observados foram significativamente diferentes dos observados nos grupos controle. Para sumarizar os efeitos da toxicidade foi utilizado o programa estatístico Toxstat 3.5 (West \& Gulley, 1996). Para amostras de efluentes e cloreto de sódio foi aplicado o teste de Steel Many One $(\mathrm{p}=0,05)$. Para amostras ambientais foi utilizado o método de Kruskal - Wallis $(p=0,05)$.

Sempre que possível, os seguintes dados foram extraídos de cada ensaio:

Concentração de efeito observado: CEO;

Concentração de efeito não observado: CENO;

Valor Crônico: VC (média geométrica entre os valores de CEO e CENO);

Valor Crônico Estimado: VCest (concentração nominal do agente químico que não causa efeito expressivo na sobrevivência e crescimento de larvas, ao final do ensaio, calculada multiplicando-se o fator 0,3 pela $\mathrm{CL}_{15} ; 168 \mathrm{~h}$ ), definido na ABNT-NBR 15499/2007 (ABNT, 2007);

Concentração Letal: $\mathrm{CL}_{15}$, calculado com o pelo método de interpolação linear (ICp).
O grupo de amostras com efeitos sobre a sobrevivência e sobre o crescimento foi analisado comparando-se os resultados do VCest (baseado na sobrevivência) e VC (baseado nos efeitos sobre a sobrevivência e crescimento).

\section{RESULTADOS E DISCUSSÃO}

Assim como no presente trabalho, Bertoletti (2000, 2009), ao propor a metodologia para a utilização de $D$. rerio em ensaios de toxicidade crônica, também se fez valer de dados referentes ao crescimento de $P$. promelas. Segundo este autor, "considerando os efeitos tóxicos no crescimento de $P$. promelas, em ensaios de curta duração, parece razoável utilizar esse critério nos experimentos com $D$. rerio, sendo pertinente fazer extrapolações entre a sobrevivência e o crescimento com base nas características de outro ciprinídeo, tal como P. promelas".

Considerando o efeito do cloreto de sódio, ao calcular a concentração que causa a letalidade em $15 \%$ dos organismosteste $P$. promelas, obteve-se uma média da $\mathrm{CL}_{15}$ igual a $1796,1 \mathrm{mg} \mathrm{L}^{-1}$ com um coeficiente de variação (CV) de 0,04\%. Bertoletti (2000), em ensaios ecotoxicológicos com $D$. rerio na fase larval (Tabela 1) obteve uma $\mathrm{CL}_{15}$ de $2000,0 \mathrm{mg} \mathrm{L}^{-1}$ para o cloreto de sódio $(\mathrm{n}=5)$.

Assim, quando estimados para a metodologia da ABNT (VCest), os valores crônicos encontrados neste trabalho (538,4 $\mathrm{mg} \mathrm{L}^{-1}$ ) foram muito próximos aos $600 \mathrm{mg} \mathrm{L}^{-1}$ encontrados por Bertoletti (2000). Baseado nestes dados é possível concluir que, para o cloreto de sódio, $D$. rerio e $P$. promelas apresentam sensibilidade semelhante. A sensibilidade de $D$. rerio e $P$. promelas foi avaliada também em estudo realizados por Stephan et al. (1983), que observou semelhança nos resultados de ambas as espécies para substancias químicas e atribuiu ao fato das duas espécies pertencerem à mesma família Cyprinidae. O efeito sobre o crescimento dos organismos não foi observado. A ação da substância cloreto de sódio sobre os organismos pode estar diretamente relacionada somente a estresse osmótico provocando mortalidade, não sendo eficiente para analisar o efeito sobre o crescimento.

Entre as amostras de efluente avaliadas neste trabalho, $49 \%$ foram caracterizadas como tóxicas, e foram, então, divididas pelo tipo de toxicidade apresentada (Fig. 1). Segundo os dados apresentados, mesmo na ausência de efeito tóxico sobre a sobrevivência, ainda podem haver efeitos sobre o crescimento dos organismos.

Tabela 1 - Ensaio de toxicidade crônica com $\mathrm{NaCl}$. Estimativas dos efeitos crônicos para $P$. promelas e para $D$. rerio, calculadas pelo método ICp.

\begin{tabular}{lcccc}
\hline Espécie & $\begin{array}{c}\text { Média das } \\
\mathrm{CL}_{15} ; 168 \mathrm{~h}\end{array}$ & $\begin{array}{c}\text { Valor crônico estimado } \\
\text { Média das } \mathrm{CL}_{15} ; 168 \mathrm{~h} \\
\text { com fator } 0,3\end{array}$ & $\begin{array}{c}\text { Número } \\
\text { Amostral }\end{array}$ & $\mathrm{CV}$ \\
\hline $\begin{array}{l}\text { Danio rerio } \\
\begin{array}{l}\text { Pimephales } \\
\text { promelas }\end{array}\end{array}$ & $17900,0 \mathrm{mg} \mathrm{L}^{-1} \mathrm{a}$ & $600,0 \mathrm{mg} \mathrm{L}^{-1} \mathrm{a}$ & 5 & $*$ \\
\hline
\end{tabular}

* Não apresentado. CV = Coeficiente de Variação. Fonte: a) Bertoletti (2000); b)Este trabalho 


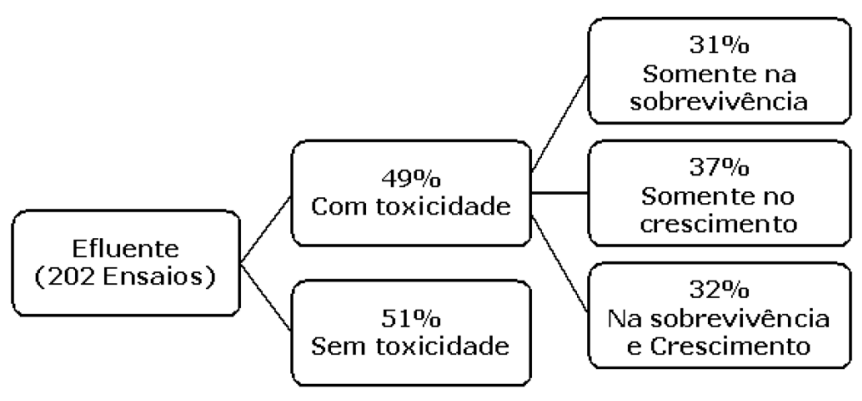

Figura 1 - Percentual de efeitos observados nos ensaios de toxicidade crônica com Pimephales promelas em amostras de efluentes industrias.

Para os ensaios crônicos com $P$. promelas em efluentes foram observados efeitos exclusivamente sobre o crescimento em $37 \%$ dos casos. Nestas amostras não houve mortalidade em nenhuma das concentrações avaliadas. Norberg-King (1989) constatou que em 59\% dos ensaios o crescimento foi o critério de avaliação mais sensível, enquanto que para a sobrevivência o mesmo ocorreu em apenas $11 \%$ dos ensaios. Pickering (1988), também avaliando ensaios com efluentes, observou que o crescimento larval foi a resposta mais sensível em $60 \%$ dos experimentos efetuados. Hughes et al. (1989) verificaram que o crescimento de Cyprinodon variegatus foi mais sensível para detectar efeitos crônicos em $52 \%$ dos efluentes ensaiados, enquanto a sobrevivência foi mais afetada somente em $7 \%$ dos ensaios."

Para amostras ambientais não é possível observar efeitos sobre a sobrevivência e o crescimento, simultaneamente. Águas subterrâneas e superficiais são submetidas aos ensaios de toxicidade como amostras integrais, não havendo diluições. Portanto, quando a amostra causa mortalidade, o efeito sobre o crescimento não pode ser observado. Desta forma, de acordo com a metodologia apresentada pela ABNT 15499:2007 no cálculo do VCest, sempre que não for apresentado efeito na sobrevivência em amostras ambientais a amostra é considerada como "Não Tóxica".

Foram avaliados 471 ensaios de toxicidade crônica de curta duração com $P$. promelas baseados em amostras de águas superficiais ou subterrâneas. Deste total, 69\% dos ensaios caracterizaram as amostras como não tóxicas. Os $31 \%$ de amostras tóxicas, foram divididos entre dois tipos de toxicidade, apresentados na Figura 2. Um percentual de 44\% das amostras ambientais consideradas tóxicas apresentaram efeitos exclusivamente sobre o crescimento dos organismosteste. Assim, mesmo que os ensaios fossem realizados com diluições, a inexistência de mortalidade impediria que o valor crônico para o crescimento fosse estimado. Novamente, para este tipo de amostra, o método perde a sua aplicabilidade.

\section{CONCLUSÃO}

A sensibilidade de D. rerio e $P$. promelas, analisando a sobrevivência para a substância de referência cloreto de sódio, mostrou-se bem próxima. Contudo, tomando como base os resultados do presente estudo, a determinação da toxicidade

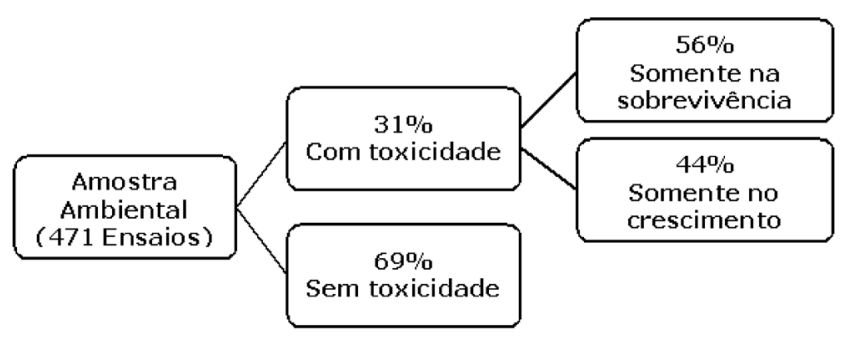

Figura 2 - Percentual de efeitos observados nos ensaios de toxicidade crônica com Pimephales promelas em amostras ambientais (águas superficiais e subterrâneas).

crônica baseada apenas nos efeitos sobre a sobrevivência dos organismos-teste proposta na NBR 15499/2007 para o peixe D. rerio pode não ser suficiente para a caracterização da toxicidade de alguns tipos de amostras.

De uma forma geral, o método baseado apenas nos efeitos sobre a sobrevivência dos organismos não permite uma boa caracterização de amostras ambientais, as quais, na maior parte dos casos, são avaliadas sem diluições, não sendo, então, possível obter a $\mathrm{CL}_{15}$ exigida pelo método para o cálculo do fator de extrapolação.

Para as amostras de efluentes industriais, mesmo o método de avaliação da toxicidade prevendo ensaios com diferentes diluições, $37 \%$ das amostras tóxicas avaliadas apresentaram efeitos exclusivamente sobre o crescimento dos organismos, durante o período de ensaio. Pela metodologia proposta na NBR 15499/2007, estas amostras seriam consideradas não tóxicas. Avaliações de substâncias químicas utilizando este método parecem não apresentar restrições, pois sempre é possível obter a $\mathrm{CL}_{15}$. Já para amostras ambientais e efluentes a estimativas dos efeitos de toxicidade crônica baseadas exclusivamente com base em resultados sobre a sobrevivência dos organismos-teste devem ser vista com cautela. Uma revisão da metodologia pela $\mathrm{ABNT}$ ou uma restrição do uso por pesquisadores e Órgãos de Fiscalização se faz necessária.

\section{REFERÊNCIAS}

ABNT (ASSOCIAÇÃO BRASILEIRA DE NORMAS TÉCNICAS). 2007. ABNT. NBR 15499: Ecotoxicologia aquática: toxicidade Crônica de curta duração: método de ensaio com peixes. Rio de Janeiro.

BERTOLETTI, E. 2009. Determinação da ecotoxicologia crônica para Danio rerio. J. Braz. Soc. Ecotoxicol., 4(1/3):1-7. http:// dx.doi.org/10.5132/jbse.2009.01.001

BERTOLETTI, E. 2000. Estimativa de efeitos tóxicos crônicos com Danio rerio (Pisces, Cyprinidae). PhD Thesis. Universidade de São Paulo, São Paulo, 102 p

HUGHES, M.M.; HERBER, M.A.; MORRISON, G.E.; SCHIMMEL, S.C. \& BERRY, W.J. 1989. An evaluation of a short-term chronic effluent toxicity test using sheepshead minnow (Cyprinodon variegatus) larvae. Environ. Pollut. 60(1-2): 1-14. http://dx.doi. org/10.1016/0269-7491(89)90216-9

NORBERG-KING, T.J. 1989. An evaluation of the fathead minnow seven-day subchronic test for estimating chronic toxicity. Environ. Toxicol. and Chem., 8: 1075-1089. http://dx.doi. 
org/10.1002/etc.5620081113

PICKERING, Q.H. 1988. Evaluation and comparison of two short-term fathead minnow tests for estimating chronic toxicity. Water Res., 7: 883-893. http://dx.doi.org/10.1016/0043-1354(88)90026-7

STEPHAN, C. E. et al. 1983. Guidelines for deriving numerical national water quality criteria for protection of aquatic life and its uses. U.S.EPA, 83p.

ZAGATTO, P.A.\& BERTOLETTI. E. 2006. Ecotoxicologia Aquática: Princípios e Aplicações. São Paulo: Ed. Rima , 464p. WEST, INC GULLEY, D. 1996. TOXSTAT®. Computer Program. Version 3.5. University of Wyoming. 